\title{
Feasibility of Infrared Thermography for Health Monitoring of Archeological Structures
}

\author{
N.M. Nandhitha ${ }^{\mathrm{a}, 1}$, S. Emalda Roslin ${ }^{\mathrm{b}}$, Rekha Chakravarthi ${ }^{\mathrm{b}}$, M.S. Sangeetha ${ }^{\mathrm{b}}$ \\ ${ }^{\mathrm{a} 1, \mathrm{~b}}$ Faculty, School of Electrical and Electronics, Sathyabama Institute of Science and \\ Technology, Old Mamallapuram Road, Chennai-600 119
}

\begin{abstract}
Archeological assets of the nation are to be preserved and rejuvenated. Ageing of these sites poses a major challenge in assessing the health of these structures. Hence it necessitates a technique that is non contact non invasive and non hazardous. Passive InfraRed Thermography is one such technique that uses an IR camera to capture the temperature variations. Thermal variations are mapped as thermographs. Interpretation of thermographs provides information about the health of the archeological structures. As the paradigm has shifted to computer aided interpretation, segmentation techniques and line profiling are used for describing the hotspot. Of the various segmentation techniques, morphological image processing provides accurate segmentation of cold spot.
\end{abstract}

Keywords: Health Monitoring, Infrared Thermography, Line Profiling, Thresholding, Morphological Image Processing.

\section{Introduction}

India is a country noted for its rich cultural heritage and every historical place is a treasure of arts and architecture, a gateway to understand the rich life style and glory of ancient kingdoms. Emperors and kings of India built temples and forts to signify their achievements and as a means of supporting spiritualism. Examples are many: Emperor Asoka built stupas all over the country to mark the important events of Gautama Buddha. In the south, Krishnadeva Raya was a great patron of arts and architecture. Likewise, Raja Raja Chola I built Bragadheeswara temple at Tanjore, Rajendra Chola I built Gangaikonda Chola puram, Pallava kings built Akamberaswarar temple at kancheepuram, rock temples at Mamallapuram to mark their victories and to spread the religion of interest.

Madambakkam in Chennai, Tamil Nadu hosts Shri Dhenupureeswarar temple built by Paranthaka Chola, the father of great Raja Raja Chola. All the pillars and walls are adorned with intricately designed sculptures to depict the happenings in Chola regime. Due to these facts, Archeological Society of India has included the

${ }^{1}$ N.M. Nandhitha, ${ }^{\text {aF }}$ aculty, School of Electrical and Electronics, Sathyabama Institute of Science and Technology, Old Mamallapuram Road, Chennai

E-mail: roemi_mich@yahoo.co.in 
temple in its database. However as years progress; it is necessary for monitoring the health of temple building. Major challenge lies in identifying an appropriate modality for acquiring images without disturbing the structures. InfraRed Thermography is one such Non-Destructive Technique is a non-contact, non-invasive and non-hazardous technique. It uses an IR camera that captures heat patterns and maps it into thermographs. These thermographs are then interpreted to determine the presence of anomalies like crack, chemical disintegration and moisture in the walls.

Brooke (2018) examined various buildings and monuments for extracting the hidden historic information present in the structures. A passive infrared thermal camera was used to identify the irregularity on the structures and to identify the materials used in the structures. The authors also claimed passive thermal infrared imaging as the best tool for evaluating the archeological structures [1]. Adamopoulos et al (2020), used thermal camera and a RGB camera for modeling of $3 \mathrm{D}$ thermograph for evaluating architectural heritage assets. The accuracy of the proposed model rely on the RGB images acquired by the high resolution camera and not on the IRT images RGB images acquired by the high resolution camera and not on the IRT images [2]. Jia-Hao He et al (2020), proposed a IRT measurement system for Structural health monitoring and compared the results obtained with that using accelerometers. They also proposed Mode Shape Recombination Method (MSRM) to perceive various large-scale structural measurements. The proposed MSRM was suitable for examining the structures in any harsh environment [3]. Image Processing algorithms along with the acquired thermographs enable us to accurately predict the hidden information in the structures, condition monitoring of electrical equipment's etc. [4][5][6].In this paper, feasibility of image processing techniques for the segmentation of anomalies in thermographs is studied. Section 2 deals with research database. In section 3, interpretation of thermographs using line profiling is dealt. Thresholding techniques used for the segmentation of anomalies from hotspots are described in section 4 . Section 5 concludes the work and provides the future directions.

\section{Research Database}

Thermographs are acquired using FLIR T335 IR camera. Defects namely crack, decay due to salt formation, and rising dampness are considered in this work. Thermographs and visible images depicting the above three conditions are shown in Figures 1-3. Thermal images are acquired from Dhenupureeswarar temple, Tiger caves and Mamallapuram rock temple.
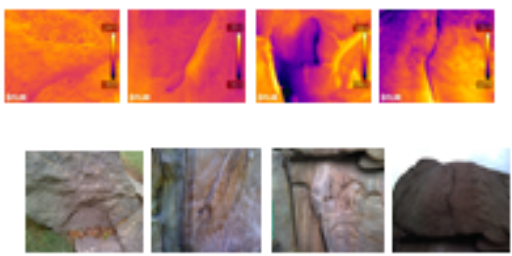

Figure.1. Thermographs and visible images depicting crack
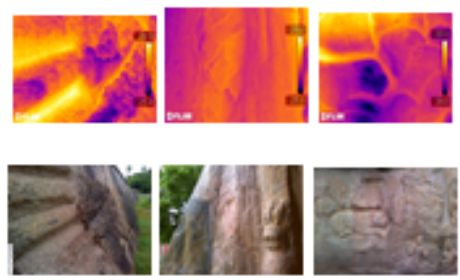

Figure.2. Thermographs and visible images depicting chemical integration 
Cracks can be seen in both visible and IR images (Figure 1). However temperature gradient cannot be obtained from visible images. Temperature gradient is an important measure in health monitoring of sculptures as it can be related to rate of deterioration. Chemical disintegration also leads to thermal variations as is seen in Figure 2. However moisture is not seen in visible images but is evident in thermal images. Also the position of moisture, shape of the affected region and thermal gradient can be obtained from thermal images [8].

\section{Anomaly Detection from the Line Profiles}

Whenever there is an abrupt variation in temperature then it indicates and anomaly. It is possible to detect the size of the anomaly through line profiling. Also it is possible to detect the seriousness of the anomaly from the thermal gradient. For cracks, the line profiling must be done along the row and for anomalies in a wall; the line profiling must be done along the column. The corresponding line profiles are shown in Figures 4 and 5.
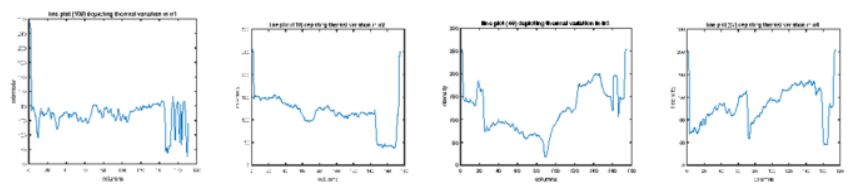

Figure. 3. Line plots depicting the intensity along the columns of a particular row for thermographs IR 1-4 depicting crack
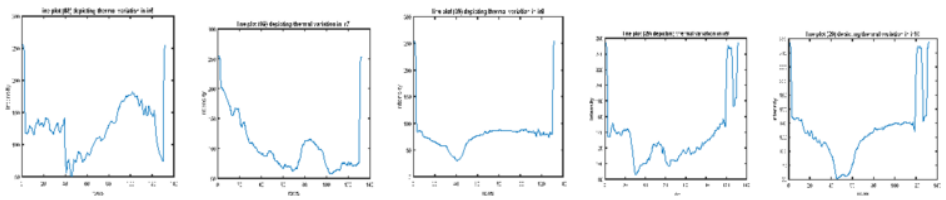

Figure. 4. Line plots depicting the intensity along the rows of a particular column for thermographs IRs 5, 6-10 depicting defects along the wall

In the above thermographs (Figure 4) at the regions of crack, the intensity decreases when compared to the neighboring regions. In all the above line profiles, it is found that, there is a dip in the intensity curve and the slope of the curve depicts the temperature gradient and the duration of the dip determines the length of the crack. On the other hand, in case of health monitoring of walls, it is necessary to understand the line profile of a particular column along the rows. Intensity variation (dip) can be easily identified and the slope and duration of the dip indicates temperature gradient and the length of the affected region.In both the cases, the slope of thermal gradient and the duration of the anomaly can be identified only for the particular row or column. It is difficult to identify all the rows/columns and to consolidate the slope and the gradient. Hence it is necessary to segment the anomaly and hence the complete information about the anomaly can be obtained. 


\section{Segmentation Techniques for Hotspot Isolation in Thermographs}

Segmentation is a subjective process that is used for identifying the Region of Interest. Of the various segmentation techniques, thresholding is the simplest method used for the isolation of cold spots from thermographs. In this technique, thresholds are fixed to reflect the Region of Interest and all the pixels with the threshold region are retained. After subjective analysis of thermographs, threshold used for extracting the low temperature region is from 110 to 120 . The gray scale thermograph, output thermographs extracted from the original thermographs are shown in Figure 5.
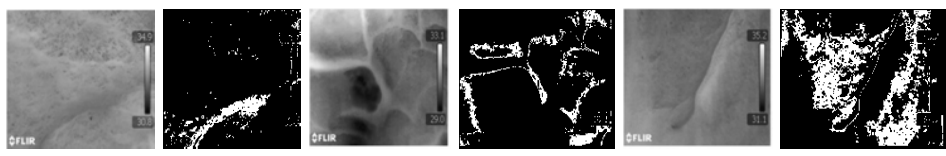

Figure. 5. Gray Scale and hotspot isolated thermographs for three sets of thermographs

From the subjective analysis, it is found that global thresholding, does not provide the desired results for the second and third set of thermographs. Hence local thresholding is needed for extracting the desired hotspots. The minimum, maximum and hotspot temperature in degree centigrade are shown in Table 1. The hotspot temperature is obtained using the formula proposed by using Equation 1 Bilodeau (2011).

Table 1. Average intensity, minimum, maximum and cold spot temperature in degree centigrade ( single threshold)

\begin{tabular}{cccc}
\hline $\begin{array}{c}\text { Average } \\
\text { intensity }\end{array}$ & $\begin{array}{c}\text { Minimum } \\
\text { temperature in } \\
\text { degree centigrade }\end{array}$ & $\begin{array}{c}\text { Maximum } \\
\text { temperature in degree } \\
\text { centigrade }\end{array}$ & $\begin{array}{c}\text { Cold spot temperature in } \\
\text { degree centigrade }\end{array}$ \\
\hline 7.128156 & 30.8 & 34.9 & 30.91461 \\
28.99658 & 31.1 & 35.2 & 31.56622 \\
8.81671 & 29.4 & 33.4 & 29.5383 \\
9.220087 & 27.9 & 31.9 & 28.04463 \\
8.891818 & 25.4 & 29.9 & 25.55691 \\
19.43978 & 25.7 & 29.8 & 26.01256 \\
11.21693 & 29 & 33.1 & 29.18035 \\
5.037792 & 29.7 & 53.3 & 30.16624 \\
18.66143 & 29.4 & 33.4 & 29.69273 \\
9.280346 & 30.3 & 34.3 & 30.44557 \\
\hline
\end{tabular}

As the cold spot cannot be identified to its full extent and undesirable regions are also found, accuracy of cold spot temperature prediction must be increased. In order to increase the accuracy, it is necessary to identify the threshold for individual thermographs or group of thermographs. Threshold, gray scale thermographs and cold spot isolated thermographs are shown in Table 2. 
Table 2. Image based thresholding for segmentation of cold spots from thermal images

\begin{tabular}{lll}
$\begin{array}{c}\text { Thermo- } \\
\text { graphs }\end{array}$ & Intensity & $\begin{array}{c}\text { Gray scale } \\
\text { thermograph }\end{array}$ \\
\hline ir_6 & $65<=$ threshold $<=100$ \\
ir_7 & $65<=$ threshold $<=74$ \\
ir_8 & $35<=$ threshold $<=51$ \\
ir_9 & $60<=$ threshold $<=97$ \\
ir_10 & $60<=$ threshold $<=97$
\end{tabular}

From the last column of the Table 2, it is found that undesirable regions are present. However the cold spots are identified to the best extent when compared to the previous thermographs. It is visible in Table 3 from the better prediction of cold spot temperature when compared to the previous technique. However undesirable regions are also present and hence the cold spot temperature must be obtained more accurately.

Table 3. Average intensity, minimum, maximum and cold spot temperature in

degree centigrade (image dependent threshold)

\begin{tabular}{cccc}
\hline $\begin{array}{c}\text { Average } \\
\text { intensity }\end{array}$ & $\begin{array}{c}\text { Minimum temperature in } \\
\text { degree centigrade }\end{array}$ & $\begin{array}{c}\text { Maximum temperature } \\
\text { in degree centigrade }\end{array}$ & $\begin{array}{c}\text { Cold spot temperature } \\
\text { in degree centigrade }\end{array}$ \\
\hline 10.2259 & 30.8 & 34.9 & 30.9644 \\
9.0579 & 31.1 & 35.2 & 31.2456 \\
0.7900 & 29.4 & 33.4 & 29.4123 \\
11.2329 & 27.9 & 31.9 & 28.0762 \\
14.1463 & 25.4 & 29.9 & 25.6496 \\
15.7641 & 25.7 & 29.8 & 25.9534 \\
3.0385 & 29 & 33.1 & 29.0488 \\
3.2823 & 29.7 & 53.3 & 30.0037 \\
5.8306 & 29.4 & 33.4 & 29.4914 \\
7.9052 & 30.3 & 34.3 & 30.4240 \\
\hline
\end{tabular}

In order to remove the undesirable regions, morphological image processing algorithm can be used. Image dependent threshold is chosen for thermograph and erosion is performed to remove the undesirable region. The size and shape of the structuring element is also dependent on the thermograph. The threshold, structuring element, its shape and size, segmented image and output image after erosion are shown in Table 4. 
Table 4. Morphological Image Processing for the accurate segmentation of cold spots from thermal images

\begin{tabular}{lccccc}
\hline Image & \multicolumn{1}{c}{ Threshold } & $\begin{array}{c}\text { Structuring } \\
\text { element/size }\end{array}$ & Segmented image & $\begin{array}{c}\text { Output image after } \\
\text { erosion }\end{array}$ \\
ir_6 & $65<=$ threshold $<=100$ & Line/1/90degrees & Line/1/90degrees \\
ir_7 & $65<=$ threshold $<=74$ & NA \\
ir_8 & $35<=$ threshold $<=51$ & NA &
\end{tabular}

Undesirable regions are removed completely as is evident from the last column of the Table 4. The cold spot temperature is identified and is shown in Table 5. The obtained cold spot temperature is accurate and hence is better than the previous two techniques. The average intensity, maximum, minimum and cold spot temperature.

Table 5. Average intensity, minimum, maximum and cold spot temperature in degree centigrade (Morphological image processing)

\begin{tabular}{cccc}
\hline $\begin{array}{c}\text { Average } \\
\text { intensity }\end{array}$ & $\begin{array}{c}\text { Minimum temperature in } \\
\text { degree centigrade }\end{array}$ & $\begin{array}{c}\text { Maximum temperature in } \\
\text { degree centigrade }\end{array}$ & $\begin{array}{c}\text { Cold spot } \\
\text { temperature in } \\
\text { degree centigrade }\end{array}$ \\
\hline 3.4396 & 30.8 & 34.9 & 30.8553 \\
1.0491 & 31.1 & 35.2 & 31.1168 \\
0.1139 & 29.4 & 33.4 & 29.4017 \\
1.2782 & 27.9 & 31.9 & 27.9200 \\
11.1670 & 25.4 & 29.9 & 25.5970 \\
13.4173 & 25.7 & 29.8 & 25.9157 \\
0.4429 & 29 & 33.1 & 29.0071 \\
0.7575 & 29.7 & 53.3 & 29.7701 \\
2.9512 & 29.4 & 33.4 & 29.4462 \\
5.7874 & 30.3 & 34.3 & 30.3907 \\
\hline
\end{tabular}

Impact of segmentation technique on identifying the RoI temperature is shown in Figure 6. 


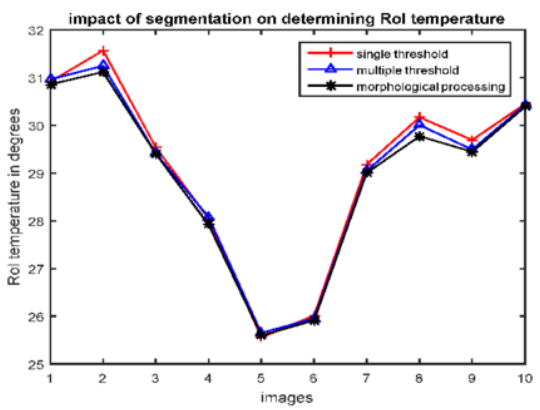

Figure. 6. Impact of segmentation technique

\section{Conclusion and Future Work}

In this work, InfraRed Thermography is used for acquiring thermal images for health monitoring of temples, tiger cave and Mamallapuram rock temple. Temperature profile and the Region of Interest are determined using line profiling and segmentation techniques. When the size of the cold spot is less and is of regular shape, then line profiling can be used for describing the cold spot. In that case, a set of few line profiles can be used for describing the cold spot. On the other hand, when the cold spot is irregular and is large in size then segmentation techniques must be used. Of the various segmentation techniques, morphological image processing technique provides accurate segmentation as it removes the undesirable regions completely. In future, semantic segmentation using deep learning techniques can be used. Also in addition to hotspot temperature thermal gradient can also be determined.

\section{References}

[1] Christopher Brooke. Thermal Imaging for the Archaeological Investigation of Historic Buildings. Remote Sensing 2018.10, 1401; doi:10.3390/rs10091401.

[2] Efstathios Adamopoulos , Monica Volinia , Mario Girotto and Fulvio Rinaudo .Three-Dimensional Thermal Mapping from IRT Images for Rapid Architectural Heritage NDT. Buildings 2020, 10, 187; doi:10.3390/buildings 10100187.

[3] Jia-Hao He, Ding-Peng Liu , Cheng-Hsien Chung and Hsin-Haou Huang. Infrared Thermography Measurement for Vibration-Based Structural Health Monitoring in Low-Visibility Harsh Environments. Sensors 2020, 20, 7067.

[4] Sudheera, K., Nandhitha, N.M., Sai, V.P.B.V. Deep Learning Techniques for Flaw Characterization in Weld Pieces from Ultrasonic Signals .Russian Journal of Nondestructive Testing, 2020. 56(10).pp. 820830 .

[5] Sangeetha, M.S., Nandhitha, N.M., Karthikeyan, S. Mathematical Relationship between Hotspot Temperature, Emissivity and Distance in Thermographs for Condition Monitoring of Electrical Equipments Venkatesh, N. Proceedings of the 2nd International Conference on Trends in Electronics and Informatics , ICOEI 2018, 2018, pp. 984-988, 8553948.

[6] Sangeetha, M.S., Nandhitha, N.M. Linear and non linear analysis of the impact of emissivity and distance on hotspot temperature in condition monitoring of electrical equipments by thermal imaging .Journal of Engineering and Applied Sciences, 2018, 13(17), pp. 7408-7413.

[7] S.Hema Kumar et.al, Effective Online Medical Appointment System. International Journal of Scientific \& Technology Research.Volume 8.Issue 09.September 2019.Pages 803 - 805. 